This is the author's copy of the publication as archived with the DLR's electronic library at http://elib.dlr.de. Please consult the original publication for citation, see e.g. https://ieeexplore.ieee.org/document/9438348

\title{
Analysis of Phase Shifts for a Rimless Wheel Rover
}

\section{J. Skibbe}

Space missions to explore Mars and Moon in situ are of high interest, but hazard terrain with steep slopes, soft soil, loose stones or high obstacles are a barrier for exploration rovers with common wheels. It was shown that rimless wheel rovers yield an enhanced performance in those environments while letting the complexity of the rover unchanged. They can cross higher obstacles, have a significant lower likelihood to get stuck and can move faster compared to common wheel rovers. At the Institute of System Dynamics and Control at the German Aerospace Center (DLR), a modular rimless wheel rover with flexible elements was designed for the purpose of planetary exploration in the above mentioned terrain. Due to the rimless wheels, the wheel circumference is not uniform. Hence, for an improved controllability of the full rover locomotion, it is reasonable to have control over the angular position of each wheel and especially over their angular position relative to each other. In this paper, several of these phase shifts are presented and compared. A setup in which the rover is driving straight forward is chosen to compare the behavior and performance of the different wheel configurations. The roll angle as well as the vertical movement of each rover segment are measured in simulation for different rover speeds and different phase shifts. A comparison between the different phase shift schemes in the sense of their waviness is made. The phase offsets have to be adjusted by means of a controller such that disturbances do not interfere significantly. For that reason, a PI controller was implemented. The study presented in this paper shows how the locomotion performance of a rimless wheel rover with compliant spokes can be improved by a well-tuned wheel position controller and the right choice of the phase shift configuration.

\section{Copyright Notice}

(C2020 IEEE. Personal use of this material is permitted. Permission from IEEE must be obtained for all other uses, in any current or future media, including reprinting/republishing this material for advertising or promotional purposes, creating new collective works, for resale or redistribution to servers or lists, or reuse of any copyrighted component of this work in other works.

J. Skibbe, "Analysis of Phase Shifts for a Rimless Wheel Rover," 2021 IEEE Aerospace Conference (50100), 2021, pp. 1-7, doi: 10.1109/AERO50100.2021.9438348. 


\title{
Analysis of Phase Shifts for a Rimless Wheel Rover
}

\author{
Juliane Skibbe \\ German Aerospace Center (DLR) \\ Münchener Str. 20 \\ 82234 Weßling, Germany \\ Juliane.Skibbe@dlr.de
}

\begin{abstract}
Space missions to explore Mars and Moon in situ are of high interest, but hazard terrain with steep slopes, soft soil, loose stones or high obstacles are a barrier for exploration rovers with common wheels. It was shown that rimless wheel rovers yield an enhanced performance in those environments while letting the complexity of the rover unchanged. They can cross higher obstacles, have a significant lower likelihood to get stuck and can move faster compared to common wheel rovers. At the Institute of System Dynamics and Control at the German Aerospace Center (DLR), a modular rimless wheel rover with flexible elements was designed for the purpose of planetary exploration in the above mentioned terrain. Due to the rimless wheels, the wheel circumference is not uniform. Hence, for an improved controllability of the full rover locomotion, it is reasonable to have control over the angular position of each wheel and especially over their angular position relative to each other.

In this paper, several of these phase shifts are presented and compared. A setup in which the rover is driving straight forward is chosen to compare the behavior and performance of the different wheel configurations. The roll angle as well as the vertical movement of each rover segment are measured in simulation for different rover speeds and different phase shifts. A comparison between the different phase shift schemes in the sense of their waviness is made. The phase offsets have to be adjusted by means of a controller such that disturbances do not interfere significantly. For that reason, a PI controller was implemented. The study presented in this paper shows how the locomotion performance of a rimless wheel rover with compliant spokes can be improved by a well-tuned wheel position controller and the right choice of the phase shift configuration.
\end{abstract}

\section{TABle of Contents}

1. INTRODUCTION..............................

2. STATE OF THE ART $\ldots \ldots \ldots \ldots \ldots \ldots \ldots \ldots \ldots \ldots . . . \ldots 2$

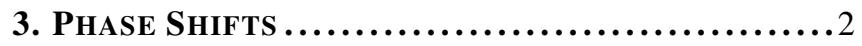

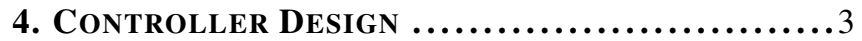

5. Simulation ................................4

6. CONCLUSION AND OUTLOOK ..................6

REFERENCES ....................................6

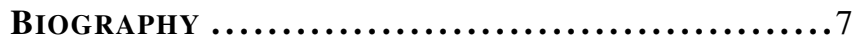

\section{INTRODUCTION}

Space missions to Mars and Moon serve to investigate surface properties, to explain the origin of these bodies and to find evidence of life via in-situ exploration. Few exploration rovers already operated on these two extraterrestrial bodies or are planned to land there in the near future, see i.a. [1]. They got through flat terrain with some slopes with a sandy and sometimes gravel surface. The Mars rover Curiosity and Perseverance also inspected craters. Even more interesting for planetary exploration are lava tubes and caves. In the case

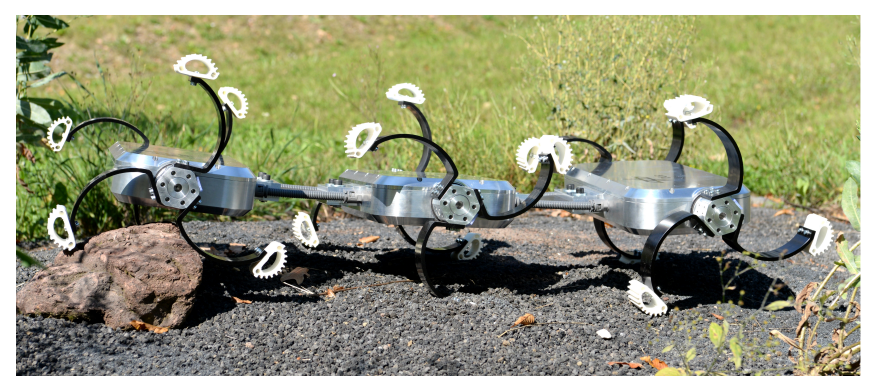

Figure 1. The scout rover developed at DLR.

that there exists life on Mars, the likelihood in these areas is assumed to be way higher than on the already explored ground [2]. These areas are known to exist from satellite pictures.

The drawback of these areas is, that they are including loose stones, high obstacles such as boulders, soft soil and additionally steep slopes. In summary, it is challenging terrain. This led to an increased research in the area of rover locomotion, in particular in the design of different wheel shapes. In space application, the goal is to maximize the performance to reach the exploration goal safely while minimizing the risks, more particularly minimizing the points of failures of a rover. Considering the locomotion system, this means that the rover can surpass as many different types of terrain as possible while having as few actuators as necessary. One concept to conciliate those requirements is the design of rimless wheels. Thereby, the advantage of only one motor per actuator compared to multiple motors in a usual leg - is combined with the benefits of the spokes of the rimless wheel: They can surmount higher obstacles compared to common wheels and they can swim through soft sand instead of sinking in [3]. Therefore, rimless wheels are a preferred choice for a rover scouting on different challenging terrain. To become an allterrain-rover, it is desirable that the rover moves efficiently on favorable, i.e. flat and rigid, ground, as well. This implies that the rover body shall move forward smoothly which may lead to:

- saving energy,

- less shaking of the rover and in particular the payload and

- better controllability and estimation of the rover path.

Such a rimless wheel rover is developed at the Institute of System Dynamics and Control at the German Aerospace Center (DLR), see figure 1 and [4]. This particular rover is taken as instance to analyze rimless wheel rover locomotion on a flat surface. It is designed out of three segments which are passively flexibly linked. At each segment, one single actuated rimless wheel with three flexible, curved spokes is mounted on the left and right side, respectively.

To reach the desired smooth locomotion on flat terrain, it is 
reasonable to drive the six individual motors not disorderly, but to control it in a certain arranged manner so that the orientation of the rimless wheels is not random. Therefore, phase shift schemes, that indicate the difference of the angular position of the wheels, are designed for a rimless wheel rover with six wheels, similar to gait schemes for legged mobile hexapod robots. A controller is implemented to adjust these phase shifts and also to keep them during driving.

In section 2, the state of the art of rimless wheel rover as well as existing gait surveys are represented. The different phase shift schemes are explained in section 3. In section 4 , the design of the controller that is adjusting the wheels is explained. In section 5, the setting of a simulation to test the performance and its results are presented. Section 6 summarizes the results and gives an outlook over the future topics of research on locomotion of rimless wheel rover.

\section{STATE OF THE ART}

The scout rover developed at DLR is not a completely new idea, several rimless wheel rovers already existed before. The RHex Robot [5] is also a hexapod rover, has compliant legs as well, but possesses only one spoke per leg. Several studies have been done about different gait schemes at the RHex robot, but they include that the wheels do not turn simultaneously, but one after another, as a result of only one spoke per wheel. In particular, a clock-driven, open loop tripod gait is studied on the RHex robot. The Lunar Whegs $^{\mathrm{TM}}$ [6] is a rimless wheel rover that is quite similar to the DLR scout rover but has no flexible spokes. It is designed to travel over different kinds of terrain on the moon, from solid ground to discrete obstacles to loose substrates, and it was tested in a simulated mars environment and a sand testbed.

Many rimless wheel rover gaits are biologically inspired. In [7], a cockroach gait serves as example for the motions of the Whegs VP rover, a rover with three-spoked rimless wheels. Comparing the cockroach gait to the Whegs VP gait, similarities, like the pitch and roll motions and the magnitude of the body motions, as well as differences like the shape of the center of mass motion are observed. Other animals are watched to switch their gaits for different speeds. Switching gaits is executed - according to [8] - rather because of dynamic stability than because of energy saving reasons. The gait change for the cockroach gait is studied by WEIHMANN and others [9] on substrates with different friction coefficients. It is observed that the cockroach changes from its tripod gait in lower speeds to a rather metachronal gait at high speeds. By comparison, the latter gait reveals a lower vertical amplitude.

Single rimless wheels are studied for less than a few decades. COLEMAN et al. [10] analyzed the three-dimensional motion of a rimless wheel under gravitational influence rolling down a slope. Many publications about the locomotion of single rimless wheels that base as well on MCGEERs studies about passive dynamic walking followed from several authors. In [11], the phase-shift control for a double-wheg module is developed, which is comparable to two rimless wheels on one segment at DLR's rimless wheel scout rover. A rimless wheel rover with adjustable angles between the spokes was studied in [12] to reduce the vertical oscillation of the rover's center of mass.

DLR's rimless wheel scout rover is developed and optimized
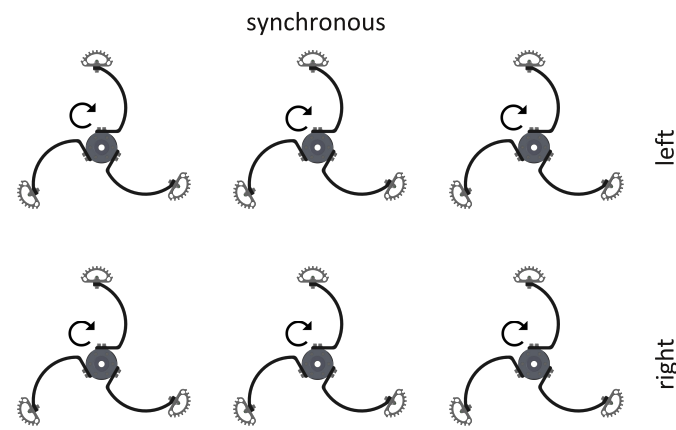

rear

middle

front

Figure 2. The synchronous phase shift scheme and the positive rotation direction of the wheels.

for its purpose to explore extraterrestrial caves and lava tubes [13]. But also scouting scenarios on earth like assistance in search and rescue tasks are conceivable. For more information about the design of the rover, see [4]. The optimal number of spokes and advantages of the current spoke shape design is studied in [3].

\section{Phase Shifts}

The shape of the rimless wheels leads to an intermittent wheel-ground-contact. In systems view, this results in a vertical rover segment movement and, depending on the relative position of the individual wheels to each other, also to a rolling movement around the longitudinal axis of each segment. To minimize these, different positions of the six rimless wheels relative to each other are investigated.

A rimless wheel with three spokes is rotationally symmetric by 120 degrees, i.e. after a rotation of $n \cdot 120^{\circ}, n \in \mathbb{Z}$, the wheel is in the same configuration as beforehand. The phase shifts will be described as a vector of length six, containing the rotation angles of the six wheels respectively, in the following order:

$$
\begin{aligned}
& \{\text { front left, front right, middle left, } \ldots \\
& \text { middle right, rear left, rear right }\}
\end{aligned}
$$

The wheel on the front left is chosen to be the reference wheel, i.e. it is always supposed to have a phase shift of 0 degrees. All the other wheels are then rotated relative to this wheel with an angle of $<120^{\circ}$ in positive direction. The positive sense of rotations is defined as the direction that a wheel has to turn to bring the rover forwards, cf. figure 2.

In the following, five different phase shift schemes will be presented. They are based on biologically inspired hexapod gaits on the one hand and the design of the rimless wheel with three spokes on the other hand. The synchronous gait is shown in figure 2, the other four phase shift schemes are depicted in figure 3.

The most obvious phase shift scheme is the one where all of the six wheels are positioned synchronously in the same orientation, see figure 2 . It can be described by $\{0,0,0,0,0,0\}$. Another phase shift scheme is the biologically inspired tripod gait. In a legged robot, the front and rear legs on one side of the body move at the same time as the middle leg 
on the respectively other side. Therefore, for the wheeled robot, the tripod phase shift scheme is designed such that the three wheels at the same positions, namely front and rear on one side and middle on the other side, are synchronous. Hence, two wheels next to each other have a phase shift of 60 degrees, what is corresponding to half the angle between two spokes. Hence, the tripod gait can be described by $\{0,60,60,0,0,60\}$. The so-called quadruped scheme has the wheel rotations $\{0,40,40,80,80,0\}$. Therefore, two wheels next to each other have a phase difference of 40 degrees. One gait was designed such that none of the wheels are synchronous. Because of the $120^{\circ}$ angle, it is reasonable to have a phase shift angle of $20^{\circ}$. To fulfil this requirement, the wheels at one segment have a phase shift of 60 degrees, and the phase shift between segments next to each other is set to 40 degrees. This phase shift scheme can be described by $\{0,60,40,100,80,20\}$ and is called "ripple" phase shift, based on the ripple gait for hexapods, presented in several publications, whereby two legs are never moving at the same time. The wave phase shift scheme is designed such that the wheels in the middle segment are always synchronous. The other four wheels have a phase shift of 40 degrees to their neighbors, respectively. Therefore, the wave phase shift scheme can be described via $\{0,80,40,40,80,0\}$.

\section{Controller Design}

The locomotion controller has two tasks: first, it adjusts the rover velocity to bring the rover to a target point and second, it has to keep the phase shifts between the different wheels. Since the wheels are not steerable, the rover has to drive curves via skid steering. Thereby, the wheels on the left side and the right side of the rover rotate with different velocities. In this case, a phase shift between the left and right wheel of a segment cannot be held. Since the main focus in this study lies in the different phase shift schemes for different velocities, the current controller is designed for straight driving only.

The control loop architecture for the full locomotion controller is depicted in figure 4 . The system constrains that a target velocity for the rover is the input to command the full rover. The wheel motors are controlled separately and expect a motor rate as input command. The general wheel velocity to move the rover forwards is computed in a feedforward. A controller will then adjust the desired phase shifts.

The rover forward velocity in general can be calculated by simple kinematic considerations. For a common wheel with radius $r$, the single wheel rate $\dot{\omega}$ for a desired rover forward velocity $v_{R}$ results from

$$
\dot{\omega}=\frac{v_{R}}{r} .
$$

Since a rimless wheel does not have a circular circumference, a reference wheel radius is introduced. Imagining the perimeter of the rimless wheel not as a circle, but as a triangle with its corners at the tips of the spokes. Then, a circular wheel with the same perimeter would have the radius

$$
r_{\mathrm{ref}}=\frac{6 l}{2 \pi} \sin \left(60^{\circ}\right)=\frac{3 \sqrt{3}}{2 \pi} l,
$$

where $l$ denotes the length from the wheel hub to the tip of a spoke. This radius is taken as the reference radius of a rimless wheel for the controller.
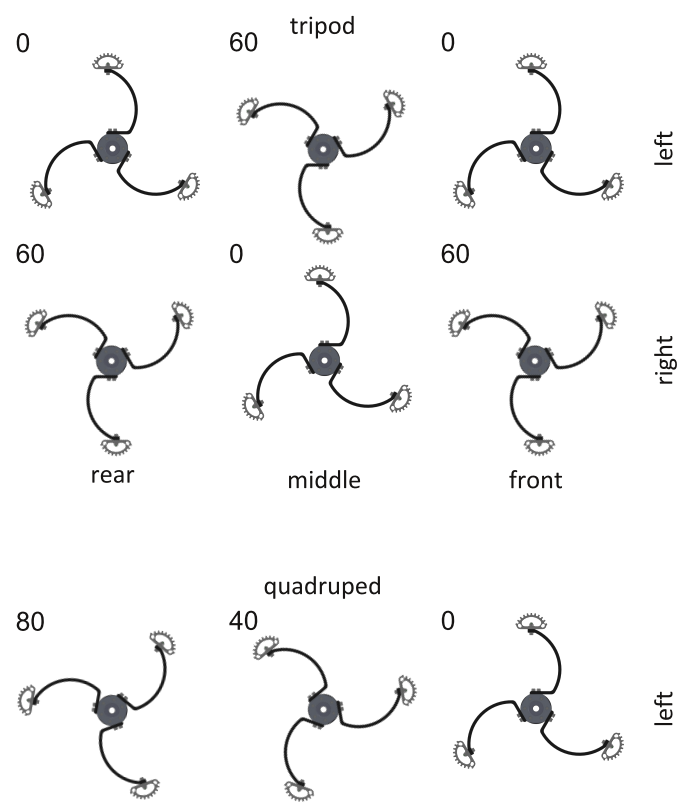

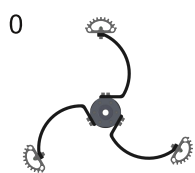

rear
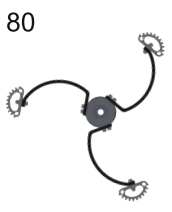

middle
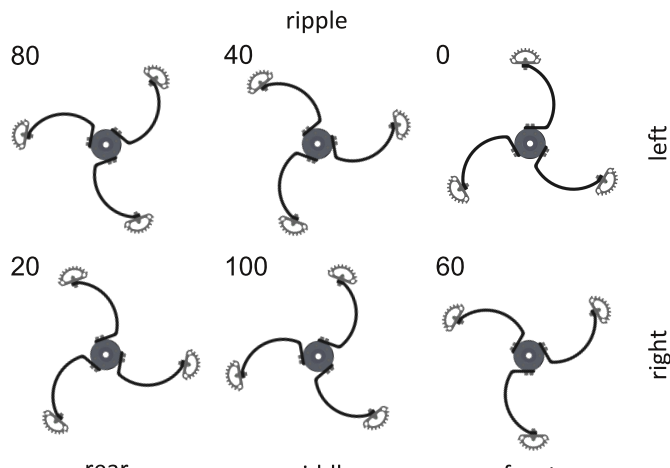

rear

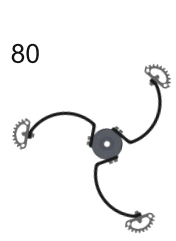

0

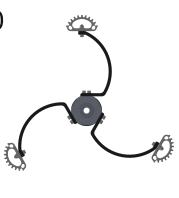

rear middle

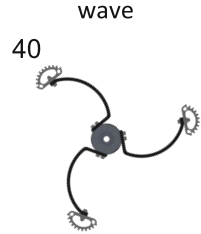

0

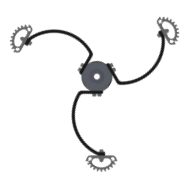

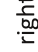

40

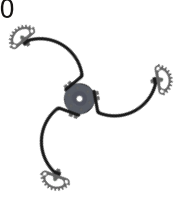

middle

front

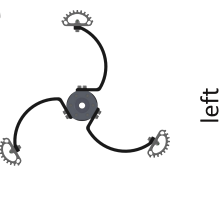

80
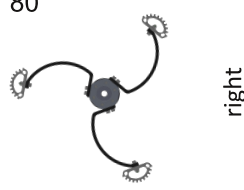

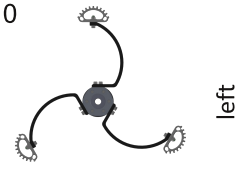

40

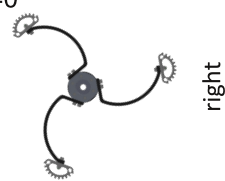

front
Figure 3. The phase shifts for the tripod, quadruped and wave scheme. 


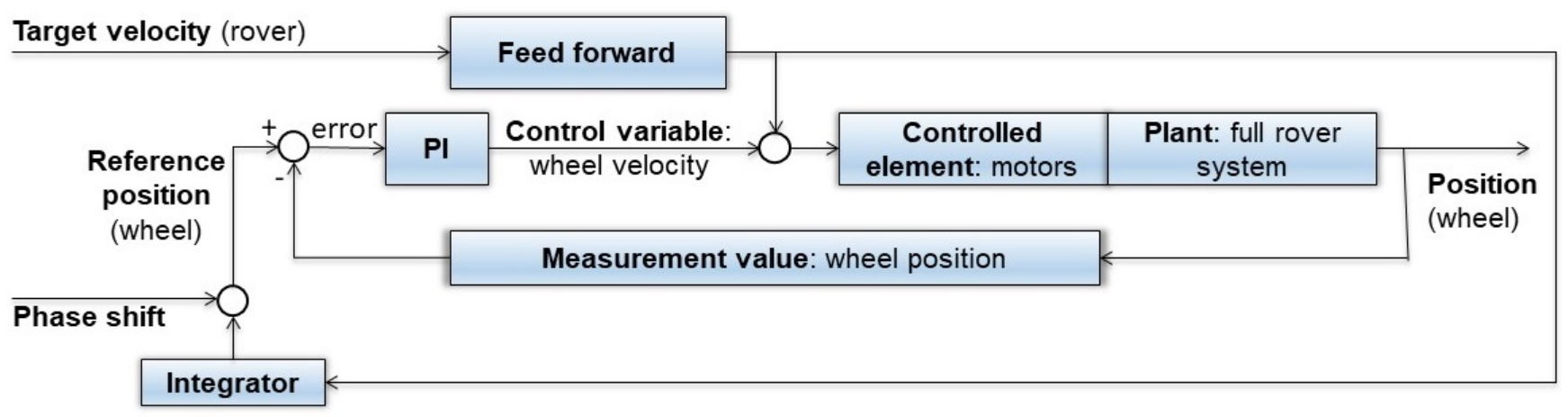

Figure 4. The control loop of the phase shift controller.

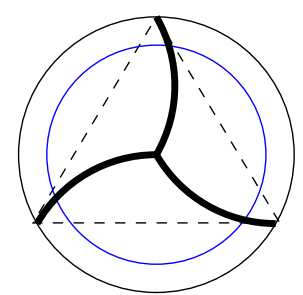

Figure 5. The circumference of the rimless wheel as circle and triangle in black, as well as the reference wheel radius in blue.

Altogether, the single wheel speed without any phase shift considerations and for a straight rover motion can now be calculated via this reference radius and equation 1 . This is done in the feedforward part of the full locomotion controller.

The output of the feedforward is integrated over time up to the current point in time $t_{0}$ and the desired phase shift $\varphi_{i}$ for wheel $i, i=1, \ldots 6$, is added to obtain the set point position $\omega_{s, i}$ for the respective wheel:

$$
\omega_{s, i}=\varphi_{i}+\int_{0}^{t_{0}} \dot{\omega}(t) \mathrm{dt} .
$$

Subtracting the actual measured wheel positions $\omega_{m, i}$ received from the motors, the wheel position error is obtained:

$$
e=\omega_{s, i}-\omega_{m, i} .
$$

Because of the symmetry of the wheels, the position error is manipulated via a modulus operation, so that the control deviation is never larger than 60 degrees:

$$
\tilde{e}=\left(\left(e+60^{\circ}\right) \bmod 120^{\circ}\right)-60^{\circ} .
$$

In this way, the correct phase shift is always reached via the shortest possible distance. A controller is then applied. Studying the system showed that a P controller is not sufficient enough, whereas a PI controller yields the adequate performance. Moreover, it was chosen due to the relations of the input and the output of the controller. The controller input is the deviation of the wheel positions and the controller output is the velocity that the respective wheels have to move with to reach the desired phase shift. The control variable is added to the output from the feed forward to obtain the final wheel velocity command to the single motors.

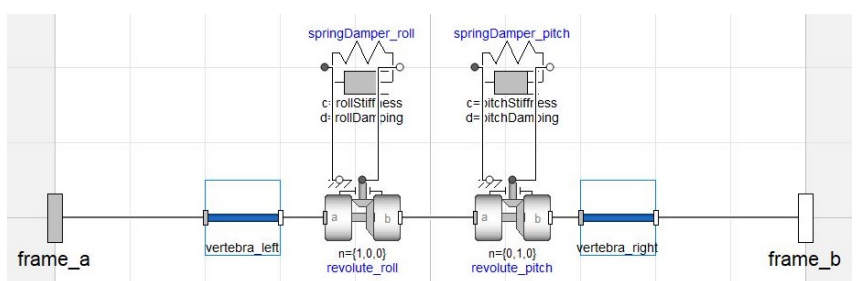

Figure 6. The vertebra model in Dymola.

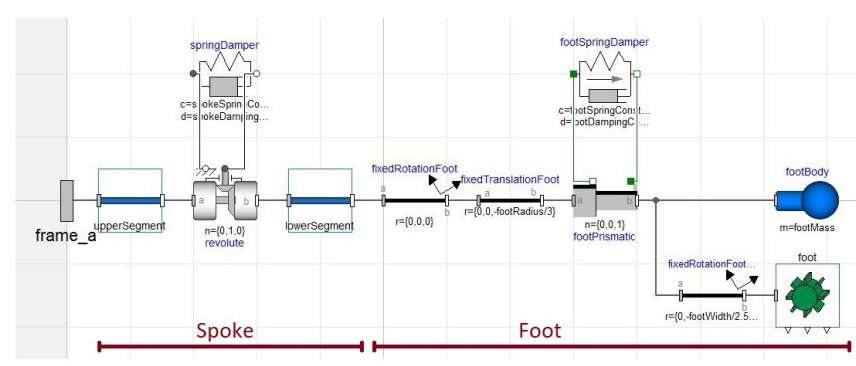

Figure 7. The model of one spoke, including the foot, in Dymola.

\section{Simulation}

The performances of the phase shifts are compared through simulation in the modeling language Modelica. The simulation model is built in Dymola in the framework of the Rover Simulation Toolkit [14] developed at DLR's Institute of System Dynamics and Control (SR).

The scout rover model has a total length of $1 \mathrm{~m}$, a width of $0.5 \mathrm{~m}$ and a weight of $19.9 \mathrm{~kg}$ under earth gravity, distributed homogeneously throughout the rover body. The flexible elements between the single segments, also called vertebra, are modeled by a spring-damper system in roll and pitch direction, see figure 6 . The stiffness in roll direction is $30 \mathrm{Nm} / \mathrm{rad}$, the stiffness in pitch direction is $10 \mathrm{Nm} / \mathrm{rad}$. The damping ratio has a value of 0.4 . The model of the spokes is shown in figure 7. The flexible, curved spokes of the rimless wheels are modeled by two rectangular beams, connected via a revolute joint with a spring-damper having a spring constant of $35 \mathrm{Nm} / \mathrm{rad}$ and a damping ratio of 0.1 . In an unstressed state, the distance from the wheel hub to the tip of a foot is $0.18 \mathrm{~m}$. For the feet, a rigid hemisphere is used as contact body, connected through a prismatic joint with linear spring damper to the spoke. The spring constant has a value of $10000 \mathrm{~N} / \mathrm{m}$ and a damping ratio of 0.2 . The contact dynamics 

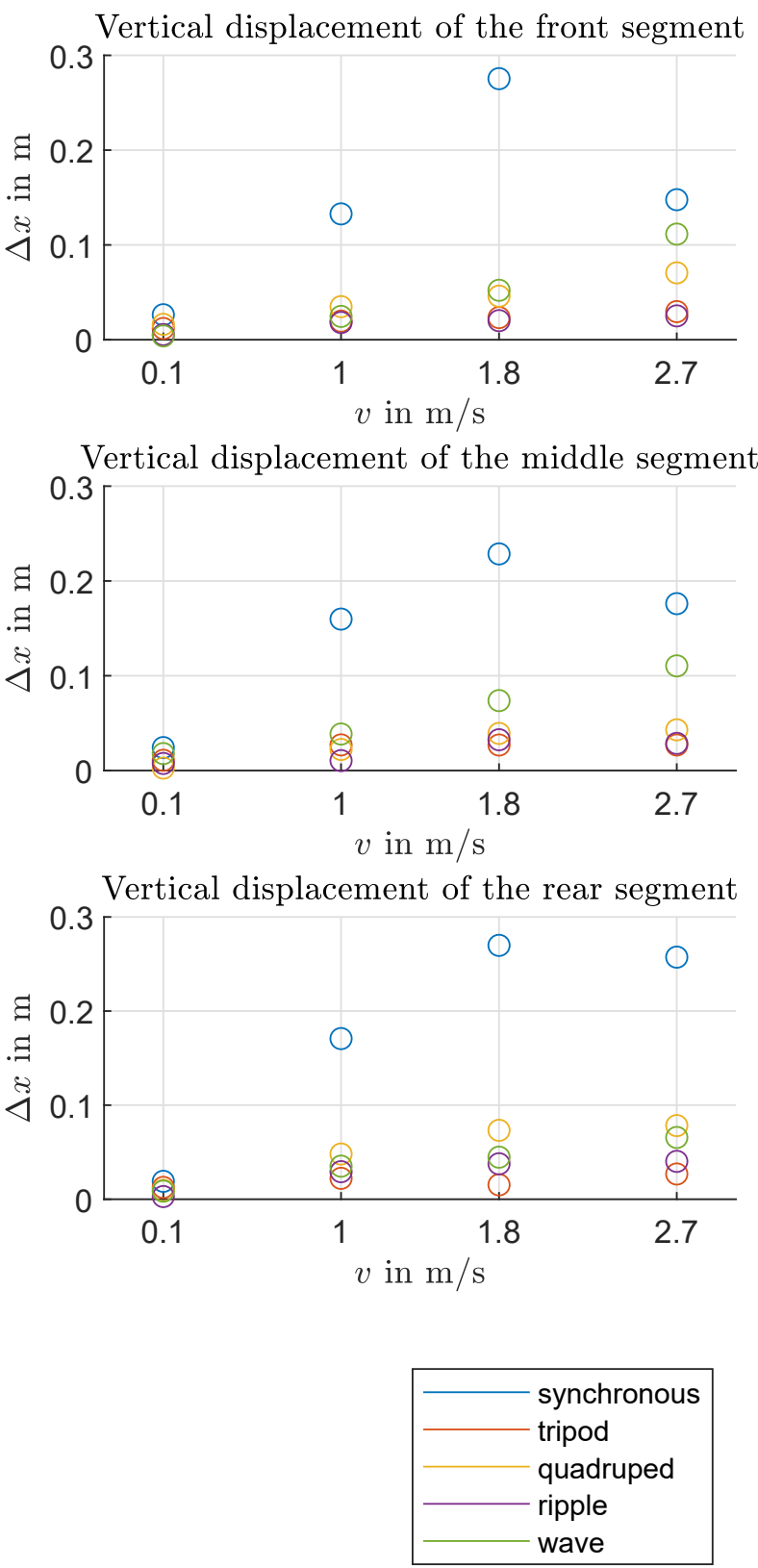

Figure 8. The vertical displacement for the three segments, the four different speeds and the five different phase angles.

between the feet and the ground are calculated following HERTZIAN theory between two hard, elastic bodies based on the indentation depth. The modulus of elasticity of the ground is $450000 \mathrm{~Pa}$ and the modulus of elasticity of the feet, which are the contact points between the rover and the ground, is $124000 \mathrm{~Pa}$. The coefficient of friction between the feet and ground is 0.55 .

In the simulation test setting, a scout rover model drives straight on a flat, hard ground under earth gravity. The scout rover is initialized with its wheels in the relevant phase shift position. Then, a constant rover velocity is commanded for 12 seconds.

\section{Vertical movement}

The vertical movement is measured for all five phase schemes at the center of each segment, which corresponds to the center of mass if the mass of the wheels and their rotation is neglected. The commanded rover speeds were $0.1 \mathrm{~m} / \mathrm{s}$, $1.0 \mathrm{~m} / \mathrm{s}, 1.8 \mathrm{~m} / \mathrm{s}$ and $2.7 \mathrm{~m} / \mathrm{s}$. The results are depicted in figure 8.

For a single rimless wheel with three spokes and a spoke length of $18 \mathrm{~cm}$, like in the simulation, the maximal vertical difference of the hub in the static case would be $9 \mathrm{~cm}$. This is the difference between the highest hub height of $18 \mathrm{~cm}$ in the case one spoke is vertical to the ground, and the lowest hub point of $9 \mathrm{~cm}$ in the case that two spokes are touching the ground as in figure 5 .

Result-The vertical movement is by far the largest within the synchronous phase shift scheme, up to $27.5 \mathrm{~cm}$ in the middle segment at a rover velocity of $1.8 \mathrm{~m} / \mathrm{s}$. This is more than three times the vertical difference of the hubs in the static case. As this rover has elastic spokes, they are leading to a prance of the rover. Visualization of the scenario is approving this behavior. Also in the wave phase shift, a negative effect of the elasticity of the spokes can be observed. It is the only other phase shift, next to the synchronous one, that has a higher vertical displacement than $9 \mathrm{~cm}$, but only for the fastest rover velocity. Especially for the wave scheme, it can be observed that the vertical displacement increases with an increasing speed. A tendency to this behavior can be seen also for the other phase shifts. This results from the flexibility and shape of the spokes: in low rover velocities, the spokes are compressed during vertical contact to the ground and lengthened at the beginning and end of their contact phase, which leads to a more regular effective wheel radius. In high rover velocities, this effect cannot arise due to inertia of the spokes. Moreover, the spokes bounce more which is lifting the rover body. In general, the ripple scheme and the tripod scheme have the best performance in terms of the vertical movement, especially in rover velocities of $1.0 \mathrm{~m} / \mathrm{s}$ and higher. The vertical displacement of the triple gait is always less than $3 \mathrm{~cm}$ for all segments in all of the measured rover velocities. Also, the ripple phase shift shows a good performance with vertical displacement of maximal $4.0 \mathrm{~cm}$ which was measured in the rear segment at the rover velocity of $2.7 \mathrm{~m} / \mathrm{s}$

\section{Roll angle}

The roll angle is measured for all the five phase schemes at each segment. The maximum absolute values of the roll angles for all of the five phase shift schemes in the four different rover velocities are depicted in figure 9.

For a single segment the maximal roll angle in the static case occurs when the wheel hub on one side of the rover is on its lowest point of $9 \mathrm{~cm}$ (cf. previous subsection) and the other wheel hub is on its highest point of $18 \mathrm{~cm}$. For the sizes like in the simulation, the maximal roll angle is given by $13.3^{\circ}$.

Result-The maximum roll angle shows a different behavior for the different phase shift schemes, especially in the middle segment. Compared to the maximal roll angle in the static case, the only two cases where the roll angle was higher was in the rear segment of the quadruped $\left(13.9^{\circ}\right)$ and ripple $\left(13.4^{\circ}\right)$ phase shift at a rover velocity of $2.7 \mathrm{~m} / \mathrm{s}$. This results again from the elasticity of the spokes, bringing oscillations into the system. 

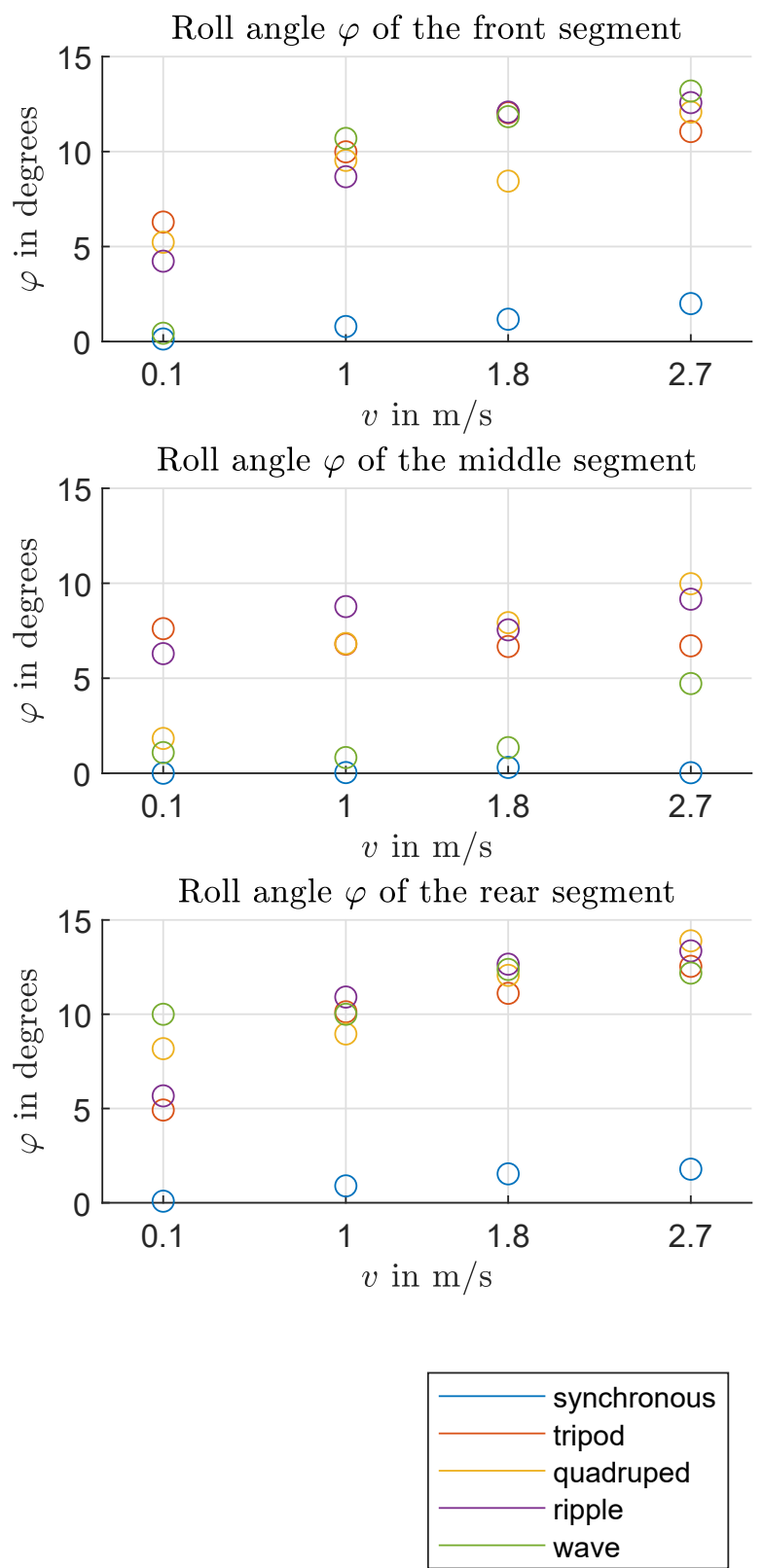

Figure 9. The roll angle for the three segments, the four different speeds and the five different phase angles.

The synchronous phase shift scheme shows almost no roll angle in all of its three segments for all of the speeds. The wave scheme has a small roll angle in its middle segment, but a roll angle of up to 13.2 degrees in its front and 12.3 degrees in its rear segment. In general, it is observable that the roll angle in the middle segment is always lower than 10 degrees, independent of the phase shift scheme and the speed. This can be explained by the fact that the neighboring segments are compensating the movement via the flexible connection between the segments. For all phase shift schemes except the synchronous one, the roll angle in high speeds is higher than 10 degrees. Moreover, it is observable that in slow speeds, the roll angle of the front segment is lower than in the rear segment for the wave and the quadruped schemes. This effect can also be seen in a lower extend in other phase shifts and rover velocities. It can be explained by a combination of the phase shift schemes and the influence of the segments on each other due to the flexible connections between them.

\section{Interpretation}

All in all, there is not one preferred phase shift scheme that exceeds all the others in performance of waviness for all speeds. The synchronous scheme is good if a small roll angle is desired, but it has a high vertical movement, except in low speeds of $0.1 \mathrm{~m} / \mathrm{s}$ : thereby, the vertical displacement of the single segments is similar to the other phase shift schemes, and therefore the synchronous scheme would be the preferable choice. The tripod scheme has an acceptable roll angle and also a low vertical movement compared to the others and would be the preferred choice for low waviness in all rover velocities and on hard ground. The quadruped phase shift scheme has lower vertical displacement than the tripod scheme in the front and middle segment, but a higher one in the rear segment. In the roll angle, it performs well in lower speeds and relatively poor especially in the middle and rear segment. The ripple scheme has a high roll angle in almost all speeds and segments. On the other side, the vertical displacement is one of the best for all segments and rover velocities. The wave scheme has the second worst vertical movement in the front and middle segment. Moreover, in the front and rear segment, it has a large roll angle compared to the other phase shift schemes.

\section{Conclusion and Outhook}

Different phase shift schemes for a rimless wheel rover were introduced and compared for locomotion on flat terrain. The synchronous scheme is the preferred choice in a very low speed, whereas the tripod gait shows the best overall performance compared to the other phase shift schemes. Moreover, a controller to adjust and keep the phase shifts was implemented.

As described in the chapter of the controller design, the current controller cannot handle skid steering. Therefore, further controller development should include an expansion for curve driving. Further investigation will also include phase shift scheme performances on different ground with variable properties, in particular sands and rough terrains. Besides, tests on hardware are planned.

\section{REFERENCES}

[1] A. Ellery, Planetary Rovers: Robotic Exploration of the Solar System. Berlin, Heidelberg: Springer Berlin Heidelberg, 2016, ch. Survey of past rover missions, pp. 59-69.

[2] A. Daga, C. Allen, M. Battler, J. Burke, I. Crawford, R. Leveille, S. Simon, and L. Tan, "Lunar and Martian Lava Tube Exploration as Part of an Overall Scientific Survey," LPI Contributions, Nov. 2009.

[3] L. Stubbig, R. Lichtenheldt, F. Becker, and K. Zimmermann, "Model-based development of a compliant locomotion system for a small scout rover," 2017.

[4] M. Wiesner, "Design and Control of a Planetary Exploration Scout for Extreme Environments Based on the Rimless Wheel," Master's thesis, Universität der Bundeswehr München, 2018.

[5] U. Saranli, M. Buehler, and D. E. Koditschek, "RHex: A Simple and Highly Mobile Hexapod Robot," The 
International Journal of Robotics Research, vol. 20, no. 7, pp. 616-631, 2001.

[6] P. A. Dunker, W. A. Lewinger, A. J. Hunt, and R. D. Quinn, "A Biologically Inspired Robot for Lunar InSitu Resource Utilization," in 2009 IEEE/RSJ International Conference on Intelligent Robots and Systems, 2009, pp. 5039-5044.

[7] R. T. Schroer, M. Boggess, R. J. Bachmann, R. Quinn, and R. Ritzmann, "Comparing cockroach and Whegs robot body motions," IEEE International Conference on Robotics and Automation, vol. 4, pp. 3288-3293, 2004.

[8] M. C. Granatosky, C. M. Bryce, J. Hanna, A. Fitzsimons, M. F. Laird, K. Stilson, C. E. Wall, and C. F. Ross, "Inter-stride variability triggers gait transitions in mammals and birds," Proceedings of the Royal Society B: Biological Sciences, vol. 285, no. 1893, 2018.

[9] T. Weihmann, P.-G. Brun, and E. Pycroft, "Speed dependent phase shifts and gait changes in cockroaches running on substrates of different slipperiness," Frontiers in Zoology, vol. 14, no. 1, p. 54, 2017.

[10] M. J. Coleman, A. Chatterjee, and A. Ruina, "Motions of a rimless spoked wheel: a simple three-dimensional system with impacts," Dynamics and Stability of Systems, vol. 12, no. 3, pp. 139-159, 1997.

[11] M. Fremerey, S. Köhring, O. Nassar, M. Schöne, K. Weinmeister, F. Becker, G. S. Đorđević, and H. Witte, "A Phase-Shifting Double-Wheg-Module for Realization of Wheg-Driven Robots," in Biomimetic and Biohybrid Systems, A. Duff, N. F. Lepora, A. Mura, T. J. Prescott, and P. F. M. J. Verschure, Eds. Cham: Springer International Publishing, 2014, pp. 97-107.

[12] O. Nassar, M. Fremerey, M. Abdelhameed, F. Tolbah, and H. Witte, "Wheg-module with electromagnetic spokes," Sep. 2014.

[13] A. F. X. Pignède and R. Lichtenheldt, "Modeling, Simulation and Optimization of the DLR Scout Rover to Enable Extraterrestrial Cave Exploration," in accepted at: The 6th Joint International Conference on Multibody System Dynamics and the 10th Asian Conference on Multibody System Dynamics, 2021.

[14] M. Hellerer, S. Barthelmes, and F. Buse, "The DLR Rover Simulation Toolkit," in 14th symposium on advanced space technologies in robotics and automation, E. van Beekhuizen, Ed. ESA's Automation and Robotics group, Jun. 2017.

\section{BIOGRAPHY}

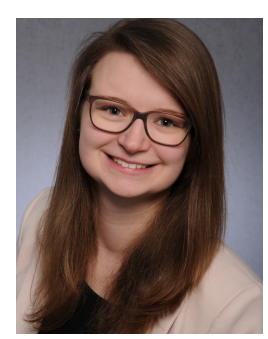

Juliane Skibbe studied Mathematics at the University of Würzburg and the Université d'Orléans. She wrote her Master's Thesis at the German Aerospace Center on the mathematical modelling of the compliant spokes for a rimless wheel rover. After finishing her Master's degree in 2018, she started working as a research associate at the Institute of System Dynamics and Control of the German Aerospace Center (DLR). Her main focus is on developing locomotion control algorithms for planetary exploration rover. 